\title{
Experiencia de gamificación para la enseñanza de un segundo idioma
}

Hugo Trejo-González

http://orcid.org/0000-0002-8703-0629

Universidad de Guadalajara, México

hugo.trejo@academicos.udg.mx

\section{Resumen}

El presente artículo presenta las experiencias generadas por el uso de la "gamificación" en el desarrollo de una materia de Francés Escrito, dentro de un programa universitario que contempla la enseñanza del francés como segunda lengua. El trabajo muestra las experiencias docentes y la perspectiva del estudiante, mediante la triangulación de las observaciones en clase y de un cuestionario sobre los aspectos esenciales de la estrategia de gamificación. Los resultados señalan que la gamificación en el aula favorece las interacciones del grupo, aumenta la participación de los estudiantes y permite atender las necesidades didácticas del objeto de enseñanza.

\section{Palabras claves (Fuente: tesauro de la Unesco)}

Aprendizaje activo; enseñanza de idiomas; enseñanza superior; gamificación en educación; teoría de juegos.

Recepción: 24/06/2020 | Envío a pares: 05/09/2020 | Aceptación por pares: 11/11/2020 | Aprobación: 09/12/2020 


\title{
Gamification Experience in Teaching a Second Language
}

\begin{abstract}
This paper presents the experiences of gamification in a French Writing Skills course as part of an undergraduate program that includes learning French as a second language. It explores teaching experiences and the student perspective by triangulating class observations and a questionnaire on the essential aspects of the gamification strategy. The results reveal that gamification in the classroom favors group interactions, increases student participation, and helps meet the didactic needs of the subject matter.
\end{abstract}

\section{Keyword (Source: Unesco Thesaurus)}

Activity learning; language instruction; higher education; gamification in education; game theory. 


\section{Experiência de gamificação para o ensino de um segundo idioma}

Resumo

Neste trabalho, são apresentadas as experiências geradas pelo uso da gamificação no desenvolvimento de uma matéria de francês escrito, de um programa universitário que abrange o ensino de francês como segunda língua. Além disso, são mostradas as experiências docentes e a perspectiva do estudante, mediante a triangulação das observações em aula e de um questionário sobre os aspectos essenciais da estratégia de gamificação. Os resultados indicam que a gamificação na sala de aula favorece as interações do grupo, aumenta a participação dos estudantes e permite atender as necessidades didáticas do objeto de ensino.

\section{Palavras-chave (Fonte: tesauro da Unesco)}

Aprendizado ativo; ensino de línguas; ensino superior; gamificação na educação; teoria dos jogos. 
La motivación en clase de lengua es un elemento esencial que favorece el interés de los estudiantes para descubrir y aprender sobre una nueva cultura. Tanto la motivación intrínseca como la extrínseca juegan un papel esencial en la implicación del estudiante en la realización o aceptación de las propuestas pedagógicas del docente. Definida como una mezcla de procesos intelectuales y psicológicos, la motivación permite que el estudiante en situación de aprendizaje particular decida sobre el esfuerzo que dedicará en la realización de tareas y actividades de enseñanza-aprendizaje (Aleksandrova, 2017, pp. 43-44).

En la enseñanza de lenguas, el trabajo de motivación en el estudiante resulta esencial para el éxito en el desarrollo de competencias lingüísticas. Los deseos, aptitudes y esfuerzos relacionados con el quehacer didáctico y de aprendizaje son indispensables tanto para el docente como para el estudiante, pues son esenciales para una práctica pedagógica positiva que permita la aceptación, asimilación y estudio de nuevas culturas (Gardner, 1985, en Castro, 2015). Para Hélou y Lantheaume (2008, p. 67), es necesario encontrar nuevos métodos, temas y actividades enfocadas en facilitar y abonar el interés de los estudiantes. Pero esto no siempre es posible, pues, aunque existan técnicas y estrategias para que los estudiantes estén más motivados en los contextos educativos, diferentes problemáticas se plantean cuando se trata de integrar nuevas propuestas didácticas que podrían favorecer el incremento o desarrollo de la motivación en clase. Del lado del profesor, la falta de tiempo, las obligaciones académicas o las formalidades administrativas conexas a la esfera profesional e institucional le impiden profundizar en técnicas o acciones didácticas para ofrecer una enseñanza en donde la motivación sea el hilo conductor de la clase (Séraphin, 2016, pp. 67-69), de tal suerte que la creación de entornos motivantes no siempre es fácil de gestionar.

En respuesta a esta problemática, diferentes metodologías apoyadas en nuevas tecnologías de la información y de la comunicación prometen facilitar el trabajo y desarrollo de la motivación en la clase. Tal es el caso de la "gamificación", metodología de enseñanza en donde la parte lúdica y el aspecto emocional y fantástico del juego son la base de las interacciones para construir entornos atractivos para los estudiantes. El objetivo principal de esta perspectiva es incluir dinámicas y características propias del juego o de los videojuegos para que los deberes o tareas considerados como pesados o en algunos casos monótonos se vuelvan interesantes y dinámicos para los implicados, a fin de aumentar su motivación intrínseca (Sánchez y Pareja, 2015, p. 3).

En relación con los contextos de enseñanza de lenguas, el hecho de abonar a la construcción de un entorno motivante para el estudiante representa una gran oportunidad para el profesor, pues reforzar positivamente los esfuerzos y resultados del estudiante de lengua ayuda a mejorar la perspectiva que tiene sobre el éxito de su práctica y esto resulta esencial para que decida continuar con su trayecto académico (Barrios, 1997, p. 26).

En consonancia con este deseo, este estudio tiene como objetivo principal mostrar las implicaciones de la estrategia didáctica de gamificación en la participación de los estudiantes en la enseñanzaaprendizaje del francés en la materia Desarrollo de la Expresión Escrita en Francés II, de la Licenciatura en Didáctica del Francés como Lengua Extranjera (LIIFLE), en la Universidad de Guadalajara. Esta intervención tiene como objetivo didáctico abonar al desarrollo de la motivación en clase para favorecer la participación de los estudiantes, cautivar su interés y mejorar su atención en el cumplimiento y la entrega de trabajos y tareas de la clase con un enfoque de enseñanza por competencias

Siendo conscientes de los riesgos y retos que implica la integración de una nueva metodología en clase en cuanto a la progresión y estructura pedagógica, este estudio es concebido para evidenciar las experiencias que resultan de la integración de 
elementos de gamificación en un curso de francés escrito. Con la ayuda de diferentes actividades y respetando las condiciones de trabajo de la Universidad de Guadalajara, hemos propuesto secuencias didácticas en donde los trabajos y tareas implicados en clase se rediseñan con base en ciertos componentes, dinámicas y mecanismos que supone la gamificación. Esto con la intención de probar y comprender los resultados y los desafíos de su implementación en clase de lengua. Por consiguiente, este trabajo muestra a grandes rasgos los cambios realizados en las secuencias didácticas, las experiencias vividas y los resultados obtenidos en un curso de lengua francesa centrado en el desarrollo de competencias escritas.

Para alcanzar nuestro objetivo, presentamos, en primer lugar, los fundamentos teóricos esenciales para comprender las características y los desafíos de la gamificación en la enseñanza. En segundo lugar, explicamos la metodología, en la que describimos el tipo de estudio, la necesidad educativa que motivó la investigación, los instrumentos utilizados, el contexto institucional y la intervención didáctica. Enseguida, presentamos los resultados del análisis de las observaciones y experiencias, así como de la percepción del estudiante. Para terminar, damos las conclusiones a las que hemos llegado.

\section{Fundamentos teóricos}

\section{Gamificación como proceso de restructuración de la enseñanza-aprendizaje}

La gamificación como estrategia pedagógica tiene como base el desarrollo de la motivación del estudiante, pero esta representa también una oportunidad para reinventar el entorno educativo, pues su integración en contextos didácticos implica: una reflexión del profesor para la actualización de contenidos, que supone un análisis de su pertinencia y una restructuración de actividades y de evaluaciones, a fin de relacionarlas asertivamente en función de las necesidades y el contexto de los estudiantes
(Oliva, 2016, p. 30). Esta perspectiva, construida a partir de elementos del juego, transfiere aspectos lúdicos y fantásticos de los videojuegos para que las actividades consideradas poco interesantes dispongan de elementos atractivos que permitan hacer frente a los problemas actuales de la educación: la falta de motivación y el compromiso escolar, con el agregado de que los componentes de dicha estrategia benefician los procesos humanos cognitivos, emocionales y sociales (Lee y Hammer, 2011, p. 3).

El hecho de integrar reglas de juego impone un desafio que implica una intervención activa del estudiante ligada a la tenacidad o perseverancia que se desarrolla en el ambiente de juego y que ayuda a que este alcance sus objetivos (Borras, 2015, p. 6). De esta manera, la construcción de un ambiente fantástico y la gloria que supone el éxito en las misiones y retos conllevan emociones detonantes que podrían ayudar a cautivar el interés de los estudiantes promoviendo el enriquecimiento de las interacciones y los debates. Para lograr esto, Diggelen (2012, en Parente, 2016, p. 13) resume diez principios esenciales de esta perspectiva: la competencia, la presión del tiempo, la escasez de elementos en alguna actividad, los puzles o problemas sin solución, la novedad de las técnicas e instrumentos, los niveles y clasificación, la presión social del grupo, el trabajo colaborativo, los premios y la posibilidad de cambio o compray, finalmente, los beneficios: dar privilegios o aumentar el poder para su uso en clase.

Por otra parte, es importante distinguir la gamificación de otras perspectivas lúdicas, como los juegos serios y el aprendizaje por el juego. En este último caso, las mecánicas son previamente establecidas en función del aspecto lúdico, en ocasiones sin considerar las intenciones del aprendizaje. Los juegos son un soporte o herramienta de apoyo para la clase (Rodríguez, 2016, p. 143) y "el objetivo principal del juego es divertir, mientras que en la gamificación el objetivo se dirige a las conductas que se desean reforzar o cambiar" (González, 2019, p. 3). En cuanto a los juegos serios o serious games, estos 
representan objetos o herramientas de aprendizaje que poseen objetivos pedagógicos y conocimientos determinados reutilizables en diferentes contextos (Sánchez, 2007, pp. 1-2). Son construidos en función de conocimientos buscados por un cierto grupo, quizás generales, y no toman en cuenta las necesidades específicas de los estudiantes y profesores. Son juegos prefabricados y "creados con objetivos específicos educativos y su fin último no es sólo divertir (como en un videojuego) sino educar" (González, 2019, p. 3).

En ocasiones mal comprendida, la gamificación es considerada como la integración de la diversión en la clase en donde lo lúdico es el objetivo final de las actividades pedagógicas. Sin embargo, la gamificación es un proceso más complejo que no está basado solamente en la diversión que suponen los videojuegos. Es importante considerar que esta perspectiva se relaciona también con la neuropsicología y que su implementación debe considerar la implicación de especialistas o formadores adecuados que puedan construir las secuencias didácticas para sacar beneficios significativos en la educación (Parente, 2016, p. 21). Según Werbach y Hunter (2012), la gamificación comprende tres grandes categorías: las dinámicas, las mecánicas y los componentes. La puesta en marcha de estos elementos consiente la creación de un entorno que puede aumentar las posibilidades didácticas de la clase. Las dinámicas representan los aspectos de base que se tienen que respetar para el funcionamiento de las actividades, como, por ejemplo, las restricciones, la narración y la progresión. Por su parte, los mecanismos y los componentes influyen en las interacciones y modos de comunicación, así como en los deseos de continuar de los estudiantes en las misiones o retos, como pasa, por ejemplo, con los premios, las recompensas y las clasificaciones en niveles (Ortiz et al., 2018, p. 4). Estos criterios deberán adaptarse a cada contexto para permitir construir el entorno ideal que pueda adaptarse a las necesidades de los estudiantes, del profesor y del contenido, pues sabemos que la institución puede condicionar los procesos educati- vos y que la introducción de elementos lúdicos no siempre se comparte en el enfoque de la educación tradicional, por lo que es importante justificar su introducción en los procesos formativos. Pensemos entonces que gamificar no implica una omisión de contenidos sino "pensar en un concepto y transformarlo en una actividad que puede tener elementos de competición, cooperación, exploración y narración y que busca la consecución de objetivos a medida de una organización" (Parente, 2016, p. 15).

Por otro lado, como el objetivo de la gamificación es atender cabalmente los objetivos de un programa educativo, es importante recordar que esta técnica puede adaptarse a una infinidad de contextos o enfoques de enseñanza. El trabajo realizado por Monguillot et al. (2015, p. 78) muestra que este enfoque didáctico mejora considerablemente la implicación del alumnado incluso en entornos educativos fuera del salón de clases. Por ejemplo, en la materia de Educación Física, en donde se ha logrado incentivar y fomentar la cultura deportiva. Por su parte, Olvera y Gutiérrez (2016, p. 212), quienes trabajaron en el ámbito de la traducción e interpretación y en el enfoque por competencias socioconstructivista, consideran que la gamificación posibilita la adquisición de conocimientos e incide positivamente en la practicidad de estos para el desarrollo profesional del estudiante. De esta manera, proyectos de ludificación del aula prometen mejoras sustanciales en desarrollo de habilidades con aplicación dentroy fuera del contexto educativo. Este enfoque muestra efectos positivos tanto en las habilidades profesionales de las materias implicadas como en las habilidades sociales que no intervienen en prácticas de enseñanza magistral: trabajo colaborativo, interacción grupal, entre otras (Aparicio et al., 2018, p. 12).

En síntesis, dado que el enfoque por competencias sugiere una convergencia entre lo social, lo psicológico, lo sensorial y lo afectivo (Argudin, 2001, en García, 2011, p. 5), la gamificación, con su influencia conductista -y siendo cuidadosos con los riesgos de los estímulos externos (Borrás, 2015, p. 6)-, pue- 
de incidir de manera positiva en lo emocional para desarrollar la motivación extrínseca y, a su vez, abrir una oportunidad para trabajar lo cognitivo y la motivación intrínseca, en el desarrollo de competencias y mediante la instauración de ambientes que alimenten el interés y el placer del estudiante por la realización de actividades (Romero et al., 2017, pp. 110-111), valiéndose de los aspectos fantásticos y las dinámicas (emociones, narración, progresión, etc.) implicados en la técnica en cuestión.

\section{Metodología}

Esta investigación tiene como objetivo evidenciar las implicaciones y los efectos de la integración de la gamificación en la participación y motivación de los estudiantes para la entrega de trabajos y tareas de la materia Desarrollo de la Expresión Escrita en Francés II de la LIDIFLE, de la Universidad de Guadalajara en México. Partiendo de un enfoque de análisis de resultados de tipo mixto, el estudio presenta, en primer lugar, la descripción de la necesidad que suscitó el interés de la intervención; enseguida, se describe el diseño de la intervención y, posteriormente, se exponen los resultados del análisis cualitativo y cuantitativo de la práctica educativa.

\section{Descripción general de la necesidad educativa}

A partir de observaciones no registradas de la materia antes mencionada, entre los años 2014 y 2018 hemos constatado que cada vez resulta más difícil que los estudiantes participen en las actividades o entreguen las tareas en tiempo y forma. Hemos podido observar a través de la impartición de clases en los diferentes semestres que en muchas ocasiones los estudiantes hacen sus entregas tarde, no se sienten motivados o lo hacen de manera obligada, solo para cumplir con la solicitud del docente o para obtener puntos para su promedio final. Como docentes titulares de la materia hemos logrado evidenciar que la motivación de los estudiantes en lo referente a la entrega de tareas o la participación en actividades en aula resulta en ocasiones difícil de gestionar, pues ellos piden la integración de actividades lúdicas o se inclinan por secuencias que integren las nuevas tecnologías. A partir de estas observaciones, hemos tratado de innovar en las prácticas docentes en la materia para ayudar a que los estudiantes se involucren de manera más activa e intrínseca en el programa curricular. Esto sin tener que recurrir a prácticas tradicionales de castigos o modificaciones de las calificaciones que obliguen al estudiante a realizar los trabajos por miedo a una nota negativa. Para esto, en primera instancia, a partir del año 2016, decidimos eliminar el valor de los trabajos y tareas de la clase del promedio final para pasar la materia. Aunque esta decisión fue aceptada positivamente por los estudiantes, no tuvo ningún efecto en su participación, pues los resultados obtenidos con esta iniciativa fueron similares o incluso inferiores a las experiencias previas, ya que, a partir de esta decisión, los estudiantes no tenían ninguna motivación extrínseca que los obligara de cierto modo a entregar sus trabajos y a participar activamente en la materia implicada. Aunque en esta ocasión no se obtuvieron resultados, esta decisión se mantuvo, pues la institución tiene como base el enfoque de enseñanza y evaluación por competencias y se consideró que quitar las tareas de la evaluación ayudaría a construir una práctica más congruente, enfocada en evaluar solamente proyectos finales que evidencien los saberes actitudinales, procedimentales y declarativos mediante la entrega de producciones contextualizadas en función de su futura realidad profesional. Dicha perspectiva didáctica ha dado resultados positivos, pues se puede apreciar mayor congruencia entre lo que promueven las tendencias educativas e institucionales en materia pedagógica y lo que sucede en el aula, para así dejar prácticas tradicionales en donde se suma una cierta cantidad de trabajos para obtener un porcentaje de la nota.

No obstante, sabiendo que el problema de la dificultad para que los estudiantes se impliquen más en los trabajos y tareas del curso era constante, decidimos en 2019 implementar la gamificación, 
enfoque que podría atender de manera precisa la motivación de los estudiantes para la realización de cualquier actividad que se considere poco interesante. Así, considerando los resultados positivos de este enfoque en otros estudios (Briceño et al., 2019; Olvera y Gutiérrez, 2016) y con el apoyo de los aportes logrados en Trejo (2019, pp. 87-91) respecto de la distinción de la características y exposición de posibilidades didácticas de las nuevas tecnologías para abonar a la estrategia lúdica en cuestión, hemos rediseñado todas las tareas y actividades de la materia para integrarlas en un enfoque pedagógico que considere nuevas dinámicas, mecánicas y componentes mediante el apoyo en herramientas digitales o con una restructuración gamificada.

\section{Instrumentos}

El análisis de los resultados de la intervención en el aula se realizó mediante la observación participante y con el apoyo de un diario de notas para registrar los efectos de la gamificación. Se rediseñaron las tareas y trabajos que apoyan el desarrollo de competencias finales de la materia y en el cuaderno de notas para cada una de las actividades se registraron las experiencias vividas. Además, se agregó un cuestionario ad hoc que permitió obtener una perspectiva general de los estudiantes. Este cuestionario ha sido diseñando mediante una escala de medición de tipo Likert y está organizado para obtener la opinión estudiantil sobre la propuesta didáctica y sobre la implicación de las tres dimensiones que constituyen el enfoque de gamificación, según Werbach y Hunter (2012): dinámicas, mecánicas y componentes. Los aspectos de cada dimensión fueron determinados con las bases de los autores mencionados, pero también con el referente teórico de Alejaldre y García (2015, pp. 76-77). Además, este instrumento fue elaborado considerando las recomendaciones de Matas (2018, pp. 44-45) para la disponibilidad de respuestas y los aportes de Hernández et al. (2014, pp. 238-243) para la elaboración de los ítems y la interpretación de resultados. Se buscó entonces realizar una triangulación de la información a partir de dos métodos de recolección implementados (Fabila et al., 2013, p. 37): observación participante y cuestionario sobre la percepción del estudiante.

\section{Descripción del contexto y de la intervención didáctica}

El grupo implicado en el estudio consta de 16 estudiantes que tomaron el curso Desarrollo de la Expresión Escrita en Francés II de la LIDIFL, cuyo objetivo principal es desarrollar las competencias escritas de los estudiantes para redactar textos de tipologías dominantes explicativas, argumentativas y prescriptivas. Para ello, el curso se divide en tres unidades de aprendizaje enfocadas en atender cada una de estas tipologías. Dicho esto, para el término de cada unidad, el estudiante deberá evidenciar sus conocimientos y competencias redactando un género discursivo de cada tipología. Para el caso particular de este estudio, se hizo la propuesta de trabajo con los géneros textuales: ensayo, para el tipo explicativo; carta de motivos, para la redacción argumentativa, y de implicación docente, como examen, reglamento o juego didáctico, para el tipo prescriptivo. Estos géneros permiten evaluar las competencias de redacción en los estudiantes, quienes deberán enfrentar situaciones de producción relacionadas con las posibles necesidades que se puedan presentar en su ámbito profesional, es decir, como docentes de lengua francesa. Cabe señalar que buscamos que dichas producciones finales se apeguen a los géneros discursivos que el futuro docente deberá integrar en su práctica profesional, considerando así una estructuración según el modelo por competencias y enseñanza situada (Díaz, 2006, pp. 25-27). A este proceso de evaluación se ciñen los trabajos y tareas que se distribuyen a lo largo de las tres unidades, que sirven para conceptualizar reglas de gramática y estructuras de los géneros textuales y ayudan a sistematizar elementos lingüísticos que fungen como entrenamiento previo del estudiante antes de las pruebas finales por competencias. 
Resulta importante mencionar que el rediseño en la entrega de trabajos y tareas es guiado a través de una propuesta gamificada que supone la integración de un modelo de compras y recompensas. Dicho modelo funciona y se mantiene activo durante todo el tiempo estipulado para la práctica educativa y es la base de la estrategia lúdica que ayuda a favorecer el interés y motivación en la entrega de trabajos y tareas. Este modelo de compras se presenta como una especie de tienda de recompensas a las que el estudiante puede acceder para hacer uso de puntos de compra generados mediante la entrega o participación oportuna en las diferentes actividades del curso (Figura 1). Esta decisión tuvo como objetivo atender algunos de los objetivos principales de la gamificación: reconocer el esfuerzo académico de los estudiantes, mejorar sus dinámicas y respuestas y garantizar mejores resultados académicos (Oliva, 2016, p. 32). Además, este esquema de recompensas permite atender elementos específicos que integran los componentes y mecánicas de la gamificación, de manera precisa en las transacciones, recompensas, puntos, desbloqueo de contenidos y gráficas sociales (Werbach y Hunter, 2012; Alejaldre y García, 2015, pp. 76-77). Esto se relaciona, según Alejaldre y García (p.78), con una gamificación estructural o profunda.

Figura 1. Tienda de premios y recompensas

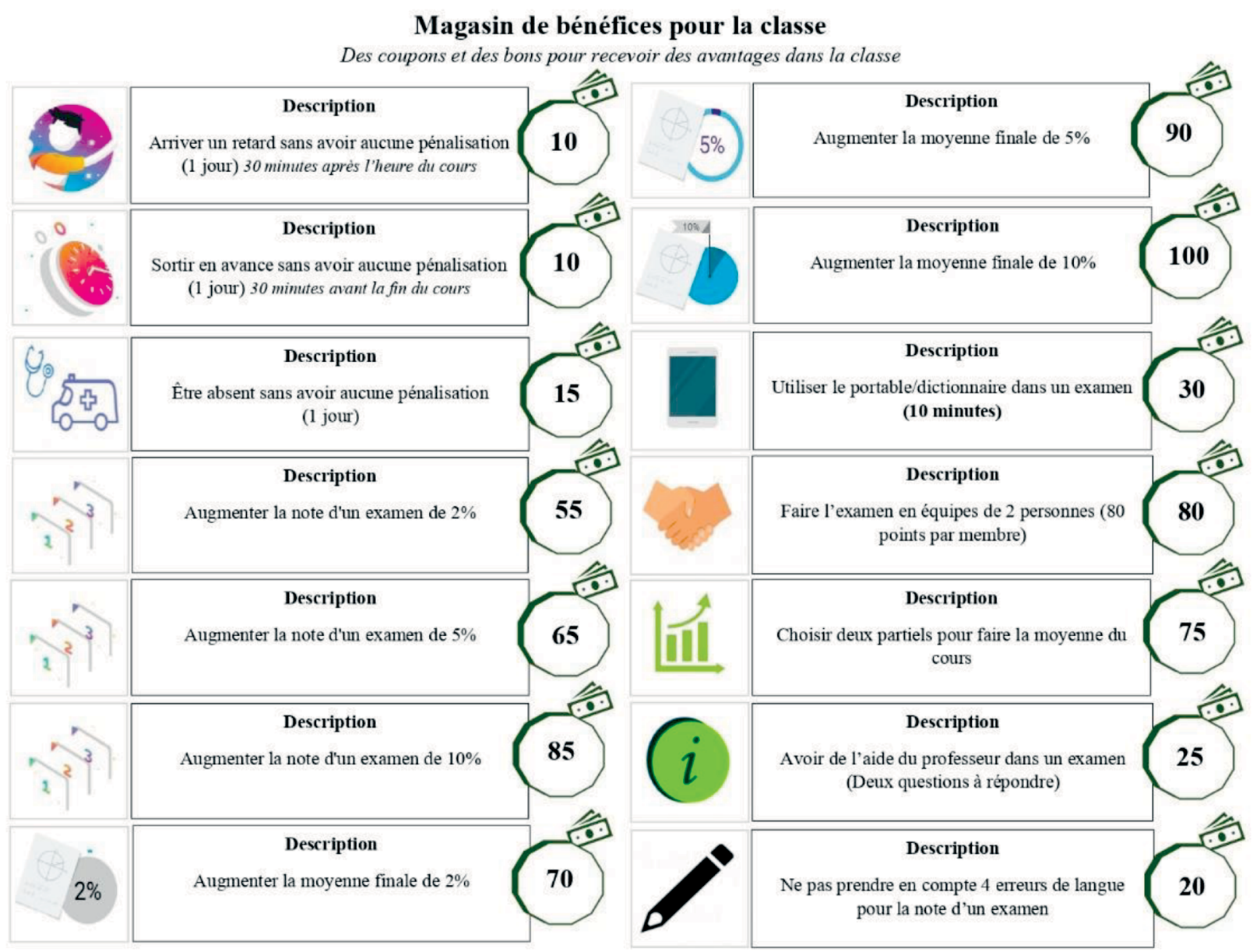

Fuente: elaboración propia. 
En la Figura 1 se puede apreciar la disponibilidad de premios y recompensas a los que el estudiante puede acceder mediante el intercambio de los puntos de compra que se obtienen a lo largo del curso con la entrega, participación y éxito en las diferentes tareas implicadas en las unidades de aprendizaje. Esta tienda de premios fue presentada al inicio del curso y su funcionamiento se explicó cuidadosamente. Se detalló que las tareas y trabajos generarían puntos para acceder a las recompensas antes mencionadas. Es importante señalar que en ningún momento se mencionó que se estaba realizado una investigación aplicada. Se buscó ante todo que el estudiante se sintiera motivado con la oferta de recompensas y que estas no afectarán considerablemente la realización de actividades, de manera que se ofrecen premios con costos variados que prometen beneficios utilizables en la clase. Por ejemplo: obtener el apoyo del profesor en un examen, utilizar el celular en una prueba, salir temprano, no ser penalizado por llegar tarde o en un examen, no tomar en cuenta un número determinado de errores. En el caso de los premios más caros, se tiene la posibilidad de hacer el examen en parejas, aumentar en un porcentaje el promedio final o elegir el resultado de dos parciales para el promedio final. Se hizo un análisis profundo para buscar no afectar el funcionamiento de clase ni disminuir las actividades y el esfuerzo. Así, cuando el estudiante tiene los puntos suficientes (casi al final del curso) para elegir el premio que permite utilizar solo dos parciales para el promedio final, se le pide que realice los tres exámenes. Además, cabe mencionar que alcanzar los premios más caros implica que el estudiante participó en todas las actividades y que ganó los primeros lugares en los concursos. Creemos entonces que un alumno que obtuvo estos logros es merecedor de los premios establecidos. Sabemos que agregar este elemento es una oportunidad para incentivar fácilmente la motivación, pero somos conscientes también de que esta práctica "puede incentivar un tipo de competencia indeseable y conducir a que el estudiante se enfoque exclusivamente en la recompensa" (Gil y Prieto, 2020, p. 119).
Por otra parte, considerando que el curso requiere de la realización de diversas tareas que ayuden al estudiante a comprender y desarrollar tanto los conocimientos declarativos como los procedimentales, la clase plantea diferentes ejercicios prácticos que buscan ayudar al estudiante en el proceso de desarrollo de competencias. Creemos que la presentación de cada una de las instrucciones de trabajo utilizadas en la clase resultaría en un trabajo extenso que dista del objetivo principal de este estudio. Por esta razón, nos enfocaremos en la descripción de 16 trabajos que sufrieron una trasformación lúdica en su realización y que son los que impactaron en el sistema de puntos y recompensas. Para ilustrar este aspecto, en la Tabla 1 exponemos la organización y rediseño de los trabajos y tareas implicados en la investigación. Además, este referente muestra algunas de las características de las transformaciones que se dieron mediante la integración de la gamificación. No obstante, esta información fue presentada a manera de resumen para facilitar su lectura y comprensión.

Como se puede apreciar en la Tabla 1, hay actividades nuevas que no existían en experiencias pasadas del curso, pero lo importante a resaltar de este descriptivo es la asignación de puntos de compra a todos los trabajos y tareas. Esto supone que todas las actividades propuestas inciden en algunos de los componentes y mecánicas de la gamificación, según Werbach y Hunter (2012): recompensas, puntos y transacciones. Es importante señalar que todas las actividades y tareas fueron explicadas en el momento de su realización. Se buscó clarificar la instrucción de cada una de ellas recordando también el impacto que tendrían en la adquisición de puntos. Tanto la tienda de premios como los puntos y clasificaciones se mostraban ocasionalmente al inicio de cada clase y se tenía acceso a estos documentos mediante la plataforma educativa Google Classroom, en donde los estudiantes podían acceder vía computadora personal o dispositivo móvil. 
Tabla 1. Organización y rediseño de actividades

\begin{tabular}{|c|c|}
\hline Tipo de actividad y número & Propuesta de actividad gamificada \\
\hline $\begin{array}{l}\text { 1. Trabajo en clase: preguntas } \\
\text { para la sistematización de } \\
\text { tipologías textuales. }\end{array}$ & $\begin{array}{l}\text { Se introdujo la herramienta Kahoot! para crear una competencia en equipos } \\
\text { con todo el grupo. Antes, solo se utilizaba un soporte escrito con preguntas } \\
\text { individuales. }\end{array}$ \\
\hline $\begin{array}{l}\text { 2. Tarea: diapositivas para la } \\
\text { sistematización de tipologías. }\end{array}$ & $\begin{array}{l}\text { Se respetó la dinámica de entrega utilizada previamente, pero en este caso la } \\
\text { actividad generaba puntos de gamificación. }\end{array}$ \\
\hline $\begin{array}{l}\text { 3. Trabajo en clase: redacción de } \\
\text { procedimientos explicativos. }\end{array}$ & $\begin{array}{l}\text { Se hizo una competencia en la que se dieron puntos a los primeros en entregar } \\
\text { sus frases. En otras ocasiones, solo se debía redactar frases con procedimientos } \\
\text { explicativos y entregarlas al profesor para su revisión. }\end{array}$ \\
\hline $\begin{array}{l}\text { 4. Trabajo en clase: clichés y } \\
\text { estereotipos frente a una } \\
\text { problemática dada. }\end{array}$ & $\begin{array}{l}\text { Se hizo una competencia en la que se dieron puntos a los primeros en entregar } \\
\text { sus opiniones. Antes, los estudiantes solo debían redactar sus frases sin límite de } \\
\text { tiempo ni en competencia. }\end{array}$ \\
\hline $\begin{array}{l}\text { 5. Trabajo en clase: dibujo sobre } \\
\text { el campo lexical, connotación y } \\
\text { denotación. }\end{array}$ & $\begin{array}{l}\text { Se desarrolló un concurso de dibujo y de redacción en relación con los temas } \\
\text { campo lexical, connotación y denotación. Esta fue una actividad nueva que no } \\
\text { existía previamente. }\end{array}$ \\
\hline $\begin{array}{l}\text { 6. Trabajo en clase: redacción } \\
\text { de ideas sobre la paridad de } \\
\text { género. }\end{array}$ & $\begin{array}{l}\text { Se hizo una competencia en la que se dieron puntos a los primeros en entregar } \\
\text { sus frases de manera pertinente. En ocasiones anteriores, los estudiantes } \\
\text { solamente redactaban las frases de manera individual, sin competencia ni } \\
\text { puntos de gamificación. }\end{array}$ \\
\hline $\begin{array}{l}\text { 7. Trabajo en clase: redacción de } \\
\text { opiniones sobre el uso excesivo } \\
\text { de las TIC. }\end{array}$ & $\begin{array}{l}\text { Se agregó mayor dinamismo a la revisión de las frases. Se abrió el debate en el } \\
\text { grupo para fomentar la discusión. Además, igual que en la actividad anterior, se } \\
\text { promovió la competencia y se agregaron restricciones. }\end{array}$ \\
\hline $\begin{array}{l}\text { 8. Trabajo en clase: revisión de } \\
\text { temas trabajados en la unidad. }\end{array}$ & $\begin{array}{l}\text { Se creó una revisión a través del uso de la herramienta Kahoot! Se mejoró } \\
\text { la dinámica gracias a los elementos de juego que la aplicación supone. } \\
\text { Anteriormente, solo se hacían preguntas orales. }\end{array}$ \\
\hline $\begin{array}{l}\text { 9. Tarea: entrega de aspectos y } \\
\text { posturas sobre el tema elegido. }\end{array}$ & $\begin{array}{l}\text { En este caso, solo se cambió la entrega vía la plataforma digital Google Classroom } \\
\text { y se agregaron los puntos de gamificación. }\end{array}$ \\
\hline $\begin{array}{l}\text { 10. Trabajo en clase: actividad } \\
\text { lúdica sobre puntos débiles y } \\
\text { fuertes de los estudiantes. }\end{array}$ & $\begin{array}{l}\text { Se realizó una actividad con todo el grupo en donde todos debieron escribir } \\
\text { algunos puntos débiles o fuertes de sus compañeros. Se fomentó el trabajo en } \\
\text { equipo y el compañerismo. Previamente, no existía esta actividad. }\end{array}$ \\
\hline $\begin{array}{l}\text { 11. Trabajo en clase: actividad } \\
\text { lúdica sobre cumplidos } \\
\text { y reconocimientos de los } \\
\text { compañeros. }\end{array}$ & $\begin{array}{l}\text { En grupo, los estudiantes debieron pensar en algo positivo que describiera } \\
\text { al estudiante que les fue asignado. El objetivo fue mejorar la comunicación e } \\
\text { integrar emociones a la clase. No existía esta actividad. }\end{array}$ \\
\hline $\begin{array}{l}\text { 12. Trabajo en clase: competencias } \\
\text { sobre abreviaciones en el } \\
\text { mundo profesional. }\end{array}$ & $\begin{array}{l}\text { Se creó una competencia colaborativa para ver qué equipo encuentra primero las } \\
\text { definiciones completas de las abreviaciones. Antes, no existía la competencia ni el } \\
\text { trabajo en equipo. }\end{array}$ \\
\hline $\begin{array}{l}\text { 13. Trabajo en clase: entrevista } \\
\text { grupal de trabajo. }\end{array}$ & $\begin{array}{l}\text { Se realizó un juego de rol en donde el docente simulaba ser el encargado de } \\
\text { recursos humanos y realizaba una entrevista de trabajo grupal. No existía esta } \\
\text { actividad en experiencias pasadas. }\end{array}$ \\
\hline $\begin{array}{l}\text { 14. Tarea: entrega de Curriculum } \\
\text { vitae en formato europeo. }\end{array}$ & $\begin{array}{l}\text { Los estudiantes utilizaron una plantilla para elaborar un CV. Se respetó el mismo } \\
\text { procedimiento de otras ocasiones, pero se otorgaron puntos de gamificación. }\end{array}$ \\
\hline $\begin{array}{l}\text { 15. Trabajo en clase: juego } \\
\text { educativo. }\end{array}$ & $\begin{array}{l}\text { Se realizó una actividad por equipos en la que se simulaba un laberinto en la } \\
\text { clase y se competía para ganar. Es un juego que no existía previamente. }\end{array}$ \\
\hline $\begin{array}{l}\text { 16. Tarea: trabajo integrador } \\
\text { individual. }\end{array}$ & $\begin{array}{l}\text { Mediante la plataforma Google Classroom, los estudiantes debían entregar los } \\
\text { avances de un trabajo de investigación de otra materia, pero que evidenciara el } \\
\text { desarrollo de competencias escritas desarrolladas en el curso. Anteriormente, no } \\
\text { existía esta actividad. }\end{array}$ \\
\hline
\end{tabular}

Fuente: elaboración propia. 


\section{Análisis}

\section{Resultados cualitativos}

En el análisis de los resultados, dos perspectivas permiten estudiar el efecto que tuvo la gamificación: a través de la observación in situ del nivel de participación e implicación de los estudiantes y a partir de los resultados de la percepción del estudiante en comparación con nuestro enfoque sobre la incidencia en las dinámicas, mecánicas y componentes propios de la intervención. Para comprender el tipo de actividades implicadas, con la información de la Tabla 1 hemos elaborado un referente de clasificación de los trabajos con el objetivo de facilitar las siguientes explicaciones (Tabla 2).

En primera instancia, considerando las experiencias en los semestres previos al año 2019, se pudo observar un aumento considerable en el nivel de implicación y participación en los trabajos de la clase. En ningún momento como docentes tuvimos que llamar la atención en clase a los estudiantes sobre el trabajo que tenían que hacer. Aunque se tuvo un mayor número de actividades tipo a y d (Tabla 2), todos los productos agregan un cierto grado de ludificación, ya que inciden en la obtención de recompensas, adquisición de puntos y clasificación en gráficas sociales que muestran el avance de los estudiantes. Dicho esto, el simple hecho de otorgar puntos de compra hizo que los estudiantes aumentaran su interés por participar en los diferentes momentos de la clase. No obstante, hubo ciertos resultados que no mejoraron considerablemente; tal es el caso de la entrega de tareas. En la Figura 2, se puede observar que las tareas corresponden al único rubro en donde no hubo cambios considerables en cuanto al nivel de participación.

Aunque solo hubo 4 tareas implicadas en la gamificación, los resultados no fueron los esperados en cuanto al nivel de participación en este rubro. Como se puede apreciar en la Figura 2, solo para la primera entrega de tarea se tuvo un nivel de participación aceptable, es decir, de 81,25\% del total de estudiantes. Aunque este número podría ser considerado como positivo, creemos que no tiene mucha relevancia en cuanto a la implicación de beneficios de gamificación, pues esta tarea fue asignada en los primeros días de clases y, desde nuestra experiencia previa, podemos decir que las primeras tareas, en general, son entregadas en tiempo y forma independientemente de la modalidad que se les asigne. En el resto de las tareas ( 9,14 y 16 en Tablas 1 y 2) se obtuvo una participación inferior al 50\% de los estudiantes (Figura 2). Desde nuestra experiencia en clase y con el apoyo de las notas de observación, consideramos que en este tipo de trabajos (tareas) no hubo un cambio positivo en cuanto al nivel de participación. Creemos que esta situación se debe a que este tipo de actividades no disponen de un grado elevado de implicación en las dinámicas, mecánicas y componentes,

Tabla 2. Clasificación de trabajos

\begin{tabular}{|l|c|c|c|c|c|c|}
\hline \multicolumn{1}{|c|}{ Tipo de trabajo } & \multicolumn{3}{c|}{ Número de la actividad } & Total \\
\hline a. Tareas & 2 & 9 & 14 & 16 & 4 \\
\hline $\begin{array}{l}\text { b. Trabajos en clase: redacción de textos o frases relativos a un tema. } \\
\text { c. Trabajo en clase: mediante la realización juegos educativos. }\end{array}$ & 3 & 4 & 6 & 7 & 12 & 5 \\
\hline $\begin{array}{l}\text { d. Trabajo en clase: mediante actividades lúdicas y dinámicas que } \\
\text { promueven la creatividad, la imaginación y la emoción. }\end{array}$ & 1 & 5 & 8 & 10 & 11 & 13 \\
\hline
\end{tabular}

Fuente: elaboración propia. 
Figura 2. Número de estudiantes que participaron en los trabajos y tareas

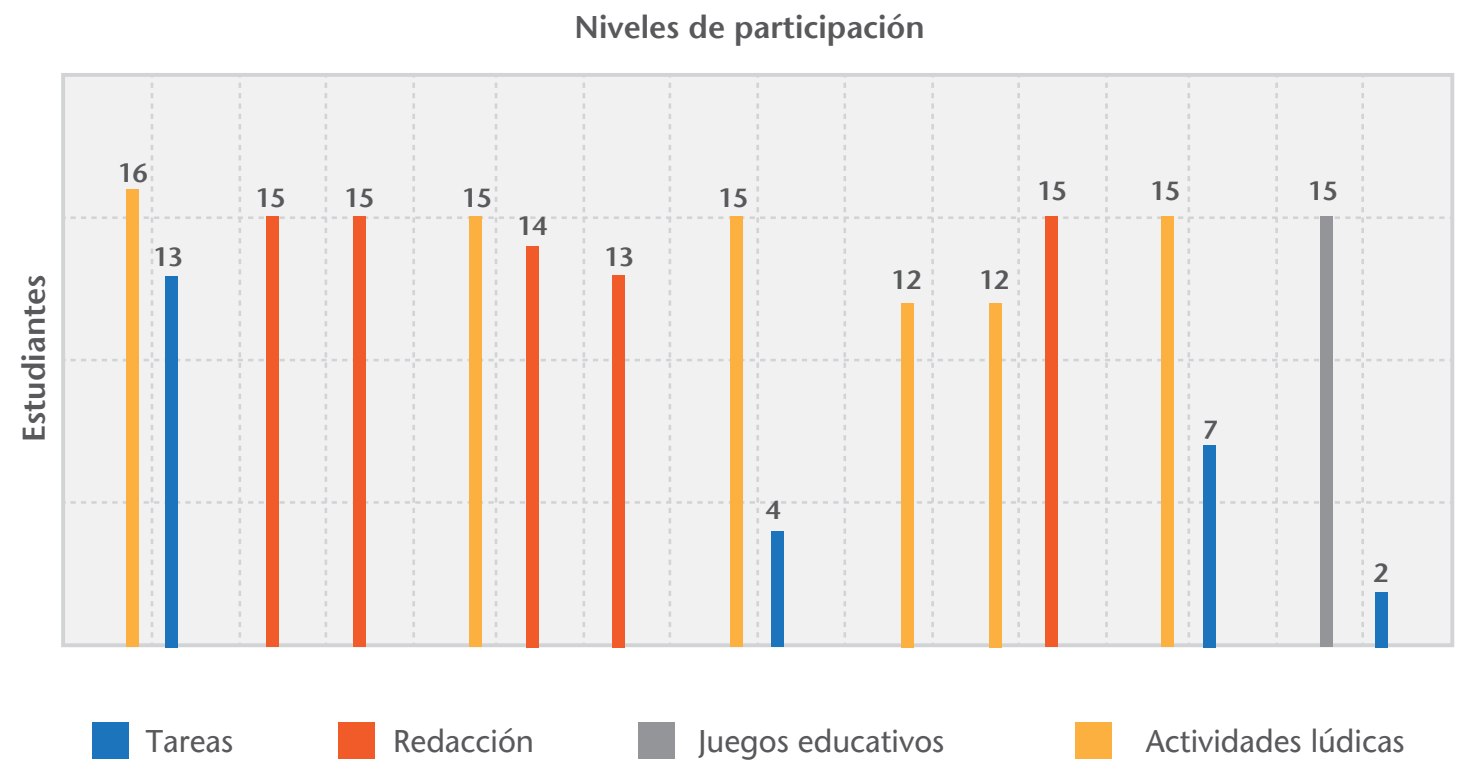

Fuente: elaboración propia.

es decir, no hubo una transformación considerable que incidiera directamente en la motivación de los estudiantes para favorecer su interés por entregar. Aunque todas las tareas otorgaban puntos de compra y el profesor recordaba en cada momento los beneficios de realizarlas, no hubo una ventaja significativa resultado de la modificación lúdica. Ante esta situación, estimamos que esto podría deberse a que, en casa, el estudiante no tiene los mismos estímulos (emociones, competencia, combate, interacciones, etc.) que le recuerden constantemente el interés por atender los objetivos, situación distinta de lo que sucedió con el resto de los trabajos en clase. Con esto, no queremos insinuar que no es posible transformar las dinámicas de las tareas sino, simplemente, que en nuestro caso las modificaciones realizadas no fueron suficientes para mejorar el nivel de participación en este rubro.

Por otra parte, en lo que se refiere a los tres tipos de trabajos restantes, se pudo observar un alto nivel de implicaciones de los estudiantes en la clase. En la mayoría de los casos, se tuvo una participación mucho más elevada que en experiencias anteriores. Como se puede observar en la Figura 2, el nivel de participación en los juegos, actividades lúdicas e incluso en las actividades de redacción fue elevado, pues en todos los casos se obtuvieron resultados superiores al 70\% de la participación total. Cabe mencionar que el porcentaje es incluso más elevado, pues durante las primeras semanas uno de los estudiantes implicados en el estudio dejó de asistir a clases por cuestiones personales, por lo que, a partir de la actividad 4, el grupo se redujo a 15 alumnos. Además, a esto se suman las ausencias de los estudiantes durante el semestre, cuestión que afectó el porcentaje de participación en las actividades que se realizaban en clase. Dicho esto, podemos afirmar que, considerando la totalidad de los estudiantes presentes en cada una de las sesiones, el 100\% participó en las actividades gamificadas. En cuanto a las observaciones precisas, podemos destacar un alto grado de disposición de los estudiantes. En todo momento, ellos estaban concentrados en terminar los trabajos en clase, pues muchas veces se competía con otros compañeros y la entrega suponía la 
obtención de premios que les servirían para mejorar sus condiciones académicas. Por su parte, en las actividades consideradas en experiencias previas como difíciles de atender (del tipo b en Tabla 2), se tuvo evidencia de una respuesta positiva por parte de la mayoría de los estudiantes. Estos trabajos en clase implican un alto grado de disposición para redactar de manera individual frases o pequeños textos sobre un tema o problemática. Esto implicaba, en experiencias previas, un esfuerzo del profesor para que las actividades fueran realizadas. No obstante, en nuestra experiencia de gamificación todos los estudiantes realizaron las cinco actividades propuestas a lo largo del semestre, esto sin tener que obligarlos a que terminaran a tiempo. Cabe subrayar que en estas actividades $(3,4,6,7$ y 12 en Tablas 1 y 2) se implementaron las siguientes características: límite de tiempo, emociones, competencia, clasificaciones, puntos y gráficas sociales.

Ahora bien, consideramos importante mencionar que, en las últimas actividades de este tipo, dos estudiantes comentaron que no les gustaba competir y estos ya no se apresuraban por ser los primeros en las clasificaciones. Esto sucedió después de haber tenido varias competencias, por ejemplo, en las actividades 6 y 12 (Tabla 1) en donde tenían que terminar primero para obtener los puntos y solo había tres lugares de clasificación. Creemos que esto se debió a que estos alumnos no habían logrado ganar en competencias anteriores, creyendo que quizás tampoco podrían ganar en las próximas a los estudiantes que habitualmente estaban en primeros lugares. Esta información pone de manifiesto que existe una gran variedad de perfiles e intereses de los jugadores (González, 2019, p. 7). Aunque esta situación solo afectó al 13,3\% del total de los estudiantes, es importante que sea considerada, pues la integración constante de competencias, concursos y combates en clase podría provocar el efecto contrario a lo esperado en los participantes.

En cuanto a las actividades pertenecientes a los grupos c y d, hemos notado un efecto positivo en la participación, actitud y percepción de los estudiantes. La implicación de estos se presentó de manera oportuna y se pudieron rescatar opiniones favorables en clase sobre este tipo de prácticas educativas. En algunos casos, comentaban que estas prácticas debían ser incluidas en todos sus cursos, pues resultaban atractivas y emocionantes. Se vieron interacciones positivas en todos los estudiantes y creemos firmemente que la relación en el grupo se fortaleció. Por ejemplo, en actividades lúdicas de tipo c, los estudiantes debían comunicar, organizarse e incluso emitir opiniones sobre los compañeros en las retroalimentaciones. Esto favoreció la comunicación entre estudiantes que no tenían contacto constante en el grupo, y aunque ello podría suceder en experiencias que no son gamificadas, el aspecto lúdico redundó en la construcción de un ambiente más relajado en donde las emociones, las sonrisas y el compañerismo se distinguieron de otras prácticas didácticas, tal como sucedió en otras experiencias del mismo tipo (Monguillot et al., 2015: 77). Por ejemplo, las competencias en la plataforma Kahoot! ( 1 y 8) fomentaron el trabajo colaborativo, la concentración y la diversión. En las actividades 5, 10, 11 y 13 se fortaleció el desarrollo de la convivencia y la comunicación mediante actividades en donde se observaba la creatividad de los compañeros y las cualidades individuales y se fomentaba la humildad y el respeto. Todas las actividades de este tipo fueron bien recibidas por los estudiantes y, además, se logró atender las necesidades precisas del programa, es decir, la sistematización y aprendizaje significativo de elementos lingüísticos para la redacción.

Por otra parte, en relación con los juegos educativos, la única actividad propuesta mostró los beneficios de esta práctica didáctica, en la que los estudiantes se divirtieron, se relajaron en el proceso y aprendieron a través de ejercicios lúdicos que potenciaron el desarrollo de competencias y habilidades sociales (Rodríguez, 2016, pp. 142-144).

Desde otra perspectiva, es importante comentar también la experiencia del docente. En cuanto a 
la restructuración y diseño de las secuencias y trabajos de la materia, podemos afirmar que el esfuerzo del docente y de sus actividades se incrementan. Consideramos que implementar esta estrategia de manera efectiva requiere, además de un interés personal, de tiempo de planeación para realizar un análisis profundo de la congruencia entre lo que se logra a través de lo lúdico y los objetivos pedagógicos que se tienen que alcanzar en la materia. Dicha tarea implica que el docente estudie y planee conscientemente la intervención sin descuidar sus obligaciones en otros cursos. En este caso, mientras sucedía la intervención de gamificación en el curso Desarrollo de la Expresión Escrita en Francés II, se impartían otras tres materias que requerían de nuestra atención. Dicho esto, el diseño y transformación de la práctica docente con la gamificación implicó tiempo y esfuerzo considerable y consideramos que no siempre se logró atender este enfoque de la manera esperada. Por ejemplo, como se puede evidenciar en los referentes previos, solo se propusieron cuatro tareas y un juego educativo. En cuanto a las tareas, esta decisión fue tomada para disminuir el trabajo docente en la revisión de estas en este curso y en los demás impartidos en su momento. Aunque no somos fervientes defensores del trabajo en casa, sí se habrían podido incluir más tareas. En relación con los juegos educativos, teníamos la intención de integrar más actividades de este tipo, pero las obligaciones docentes, académicas y de gestión no nos permitieron enriquecer más la práctica. Sin embargo, estimamos que no se afectó considerablemente nuestra práctica docente o el enfoque de enseñanza utilizado, ni el desarrollo y atención de las necesidades de la materia. Además, desde nuestra perspectiva, se tuvieron ventajas significativas a nivel general, ya que los resultados de los estudiantes fueron mejores que en experiencias anteriores y se tuvo un grado elevado de implicación y participación de todos los actores. Asimismo, la gamificación tuvo un impacto positivo en las relaciones e interacciones del grupo en el ambiente educativo sin descuidar los objetivos y sin modificar contenidos ni elementos esenciales del programa.

\section{Perspectiva docente sobre la incidencia de la gamificación en la práctica educativa:}

Con el objetivo de ilustrar el nivel de implicación que tuvo la gamificación, hemos elaborado referentes que permiten apreciar la incidencia de las diferentes actividades en las dinámicas, mecánicas y componentes. Para esto, con base en los referentes de medios digitales elaborados en Trejo (2019, pp. 87-91), hemos construido clasificaciones (Tablas 3, 4 y 5) que muestra la incidencia de la intervención didáctica en los diferentes elementos que componen la gamificación. Las casillas oscurecidas en las tablas corresponden a la presencia de los aspectos de gamificación antes mencionados en las actividades rediseñadas. Hemos simplificado la lectura de estos referentes escribiendo solamente el número de cada actividad con base en la clasificación previa en las Tablas 1 y 2.

Considerando los resultados de la clasificación elaborada a partir de nuestra observación en clase, podemos decir que hemos involucrado la mayoría de los aspectos que componen la gamificación. Desde nuestra perspectiva de análisis personal e individual de cada trabajo o tarea, creemos que hemos integrado al menos 23 de los 28 elementos que forman parte del proceso de la gamificación, según Werbach y Hunter (2012). Aunque muchos de estos aspectos no se integraron de manera constante 0 regular, nuestra propuesta pedagógica corresponde a un nivel de implicación alto en lo que se refiere a la gamificación. Cabe subrayar que en nuestra práctica gamificada no hemos logrado incidir en ciertos componentes: avatar, colecciones, combate, niveles y regalos. Esto a la luz de nuestra comprensión personal de lo que significa la intervención en cada rubro. Además, tanto las mecánicas de suerte como las dinámicas de narración fueron elementos poco trabajados en nuestra intervención. Creemos que esto es debido a que no siempre se considera una atención consciente y constante a todos los elementos que podrían aportar a la construcción de una intervención gamificada. En pocas palabras, en ocasiones 
ISSN $0123-1294$ | e-ISSN 2027-5358 | Educ.Educ. Vol. 23. No.4 | Noviembre-enero de 2021 | pp. 611-633

Universidad de La Sabana | Facultad de Educación

Tabla 3. Dinámicas de la intervención

\begin{tabular}{|c|c|c|c|c|c|c|c|c|c|c|c|c|c|c|c|c|}
\hline & \multicolumn{10}{|c|}{ Número de las actividades } \\
\hline Dinámicas & 1 & 2 & 3 & 4 & 5 & 6 & 7 & 8 & 9 & 10 & 11 & 12 & 13 & 14 & 15 & 16 \\
\hline Emociones & & & & & & & & & & & & & & & & \\
\hline Narración & & & & & & & & & & & & & & & & \\
\hline Progresión & & & & & & & & & & & & & & & & \\
\hline Relaciones & & & & & & & & & & & & & & & & \\
\hline Restricciones & & & & & & & & & & & & & & & \\
\hline
\end{tabular}

Fuente: elaboración propia.

Tabla 4. Mecánicas de la intervención

\begin{tabular}{|c|c|c|c|c|c|c|c|c|c|c|c|c|c|c|c|c|}
\hline & \multicolumn{16}{|c|}{ Número de las actividades } \\
\hline Mecánicas & 1 & 2 & 3 & 4 & 6 & 7 & 9 & 8 & 9 & 10 & 11 & 12 & 13 & 14 & 15 & 16 \\
\hline Colaboración & & & & & & & & & & & & & & & & \\
\hline Competición & & & & & & & & & & & & & & & & \\
\hline Desafios & & & & & & & & & & & & & & & & \\
\hline Recompensas & & & & & & & & & & & & & & & & \\
\hline Retroalimentaci & & & & & & & & & & & & & & & & \\
\hline Suerte & & & & & & & & & & & & & & & & \\
\hline Transacciones & & & & & & & & & & & & & & & & \\
\hline Turnos & & & & & & & & & & & & & & & & \\
\hline
\end{tabular}

Fuente: elaboración propia.

Tabla 5. Componentes de la intervención

\begin{tabular}{|c|c|c|c|c|c|c|c|c|c|c|c|c|c|c|c|c|}
\hline & \multicolumn{16}{|c|}{ Número de las actividades } \\
\hline Componentes & 1 & 2 & 3 & 4 & 5 & 6 & 7 & 8 & 9 & 10 & 11 & 12 & 13 & 14 & 15 & 16 \\
\hline \multicolumn{17}{|l|}{ Avatar } \\
\hline \multicolumn{17}{|l|}{ Colecciones } \\
\hline \multicolumn{17}{|l|}{ Combate } \\
\hline \multicolumn{17}{|c|}{ Desbloqueo de contenidos } \\
\hline \multicolumn{17}{|l|}{ Equipos } \\
\hline \multicolumn{17}{|l|}{ Gráficas sociales } \\
\hline \multicolumn{17}{|l|}{ Huevos de Pascua } \\
\hline \multicolumn{17}{|l|}{ Insignias } \\
\hline \multicolumn{17}{|l|}{ Límite de tiempo } \\
\hline \multicolumn{17}{|l|}{ Misiones } \\
\hline \multicolumn{17}{|l|}{ Niveles } \\
\hline \multicolumn{17}{|l|}{ Puntos } \\
\hline \multicolumn{17}{|l|}{ Clasificaciones } \\
\hline \multicolumn{17}{|l|}{ Regalos } \\
\hline Tutoriales & & & & & & & & & & & & & & & & \\
\hline
\end{tabular}

Fuente: elaboración propia. 
durante el diseño de actividades y secuencias didácticas resulta complicado tener en mente la complejidad y diversificación de posibilidades que esta práctica educativa posibilita y esto puede dar como resultado que el diseño gamificado se vuelva repetitivo en cuanto al tipo de actividades, ya sea porque se ven buenos resultados o porque se necesita dedicar más tiempo para diseñar propuestas con mayor diversidad de estrategias y materiales. Sin embargo, ante la gran cantidad de elementos que podrían componer una práctica de gamificación, más allá de representar una serie de pautas rigurosas que se deben respetar, la diversidad de estos rubros supone un abanico de posibilidades didácticas. El docente, al revisar todos los elementos que conforman un entorno lúdico (dinámicas, mecánicas y componentes), puede desarrollar ideas únicas que en algunos casos solo involucren algunos puntos de la gamificación. En nuestro caso, desde este enfoque, no se trató de atender rigurosamente cada uno de los elementos antes mencionados. Aunque se pudieron integrar más aspectos de la gamificación para desarrollar sus posibilidades didácticas, discurrimos que se logró experimentar ampliamente con la intervención y que esta tuvo resultados positivos en el aprendizaje de la materia implicada.

\section{Resultados cuantitativos}

\section{Perspectiva del estudiante sobre la intervención gamificada:}

Con el objetivo de enriquecer los resultados de la estrategia implementada, se aplicó un cuestionario final a los estudiantes que tenía como propósito conocer su percepción general del curso. Cabe subrayar que en ningún momento se les informó que se trataría de un estudio en donde se evaluaría la eficacia de la gamificación. Esto buscando que sus respuestas fueran lo más objetivas posible. Dicho instrumento fue elaborado en dos partes: una sección sobre perspectiva general del curso y un apartado sobre la implicación de dinámicas, mecá- nicas y componentes de gamificación. La primera sección buscó que los alumnos evaluaran las características generales del curso según su percepción general del mismo en cuanto a la metodología y a la motivación. El segundo apartado correspondía al nivel de implicación de las dinámicas, mecánicas y componentes propios de la gamificación, en donde los indicadores del cuestionario eran los mismos elementos que componen cada rubro. Para elaborar el instrumento, se utilizó la escala de Likert en ambas secciones, pero las respuestas se adecuaron a los ítems propuestos. De esta manera, en la sección sobre la percepción general de curso, se utilizó una escala de respuestas para medir la conformidad (de Totalmente en desacuerdo a Totalmente de acuerdo) y para la segunda sección se utilizó una escala de respuestas para medir el nivel de la presencia de los indicadores (de Nada a Bastante). Ambas secciones consideraron importante agregar la respuesta Sin opinión. Esto para aquellos casos en los que no se tuviera información suficiente o en donde el estudiante no comprendiera la información del cuestionario (Matas, 2018, p. 43); no obstante, cuando esta opción fue elegida por alguno, no se contabilizó en el análisis de los resultados. Además, los indicadores para la primera sección fueron redactados buscando facilitar su comprensión, es decir, sin exceder las 20 palabras (Hernández et al., 2014, p. 238). Es importante recordar que este cuestionario se aplicó para comparar la perspectiva del observador y la experiencia del estudiante y con el fin de obtener un análisis más profundo de los resultados.

\section{Indicadores utilizados en la primera sección del instrumento:}

- La metodología utilizada por tu profesor en clase tuvo una influencia positiva en el desarrollo de tus competencias.

- Hubo cambios significativos en las dinámicas propuestas por el profesor en comparación con otros cursos.

- $\quad$ Se propusieron diversas actividades lúdicas sin descuidar los objetivos específicos del curso. 
- El profesor explicó claramente el funcionamiento y la manera de trabajar en la clase con la metodología implementada.

- Las actividades durante todo el curso se dieron de manera justa y equitativa en función de las diferentes competencias de los estudiantes.

- En general, te sentiste motivado durante la realización de todas las actividades requeridas por el profesor.

- El esquema de puntos para la compra de beneficios en la clase te motiva más que el registro de tareas habitual.

Los resultados obtenidos después del vaciado de información fueron analizados con base en la propuesta de interpretación de la información sugerida por Hernández et al. (2014, pp. 238-243). En la Figura 3 y la Tabla 6, se muestra la percepción final de los estudiantes sobre la metodología, en función de los cambios significativos percibidos, de la motivación y del nivel de atención a los objetivos de aprendizaje. Dicha figura muestra el promedio general obtenido de cada estudiante en la primera sección y, además, se puede observar un resultado general del grupo en la escala de apreciación.

Figura 3. Percepción del estudiante de la metodología y la motivación

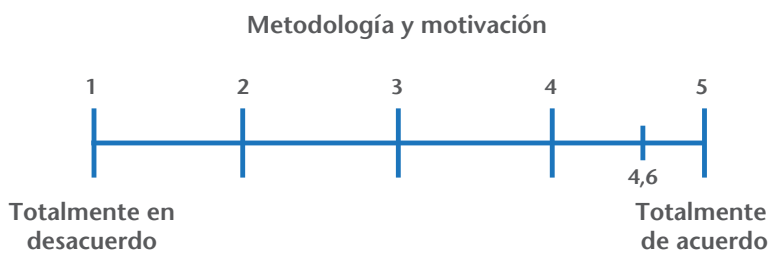

Fuente: elaboración propia.

A partir de los resultados de esta sección, podemos afirmar que tanto nuestra perspectiva como la de los estudiantes coinciden en que la gamificación tuvo un impacto significativo en la motivación general, así como en la atención de las necesidades del programa. Con un resultado de 4,6 en la escala de apreciación, podemos ver que la percepción general del estudiante se inclina hacia la aceptación total (entre las respuestas De acuerdo y Totalmente de acuerdo) en cuanto a las afirmaciones del instrumento aplicado. La postura de los estudiantes frente a la intervención es considerada como positiva, en cuanto al incremento de la motivación y en relación con la atención oportuna de las necesidades del programa. Además, en este apartado del cuestionario no se encontraron respuestas del tipo Sin opinión, por lo que podemos inferir que las afirmaciones fueron comprendidas y que el estudiante encontró evidencias para responderlo.

Tabla 6. Percepción del estudiante de la metodología y la motivación

\begin{tabular}{|c|c|c|}
\hline Metodologia y motivación & 35 & Promedio \\
\hline Estudiante 1 & 33 & 4,7 \\
\hline Estudiante 2 & 27 & 3,8 \\
\hline Estudiante 3 & 35 & 5 \\
\hline Estudiante 4 & 34 & 4,8 \\
\hline Estudiante 5 & 34 & 4,8 \\
\hline Estudiante 6 & 35 & 5 \\
\hline Estudiante 7 & 34 & 4,8 \\
\hline Estudiante 8 & 28 & 4 \\
\hline Estudiante 9 & 32 & 4,5 \\
\hline Estudiante 10 & 35 & 5 \\
\hline Estudiante 11 & 32 & 4,5 \\
\hline Estudiante 12 & 28 & 4 \\
\hline Estudiante 13 & 32 & 4,5 \\
\hline Promedio del grupo & & 4,6 \\
\hline
\end{tabular}

Fuente: elaboración propia.

En la segunda sección, siguiendo el mismo proceso de interpretación de la información, en la Figura 4 y la Tabla 7 se muestra la percepción del estudiante acerca del nivel de implicación de los elementos de la gamificación. En este caso, las tres columnas corresponden a los resultados individuales en cada uno de los rubros que supone la estrategia implementada: dinámicas, mecánicas y componentes. Para analizar los resultados, se separaron estos 
rubros a fin de comparar cada uno de manera individual con nuestra observación en clase y con los referentes creados previamente en las Tablas 3, 4 y 5.

Figura 4. Percepción del estudiante sobre la gamificación
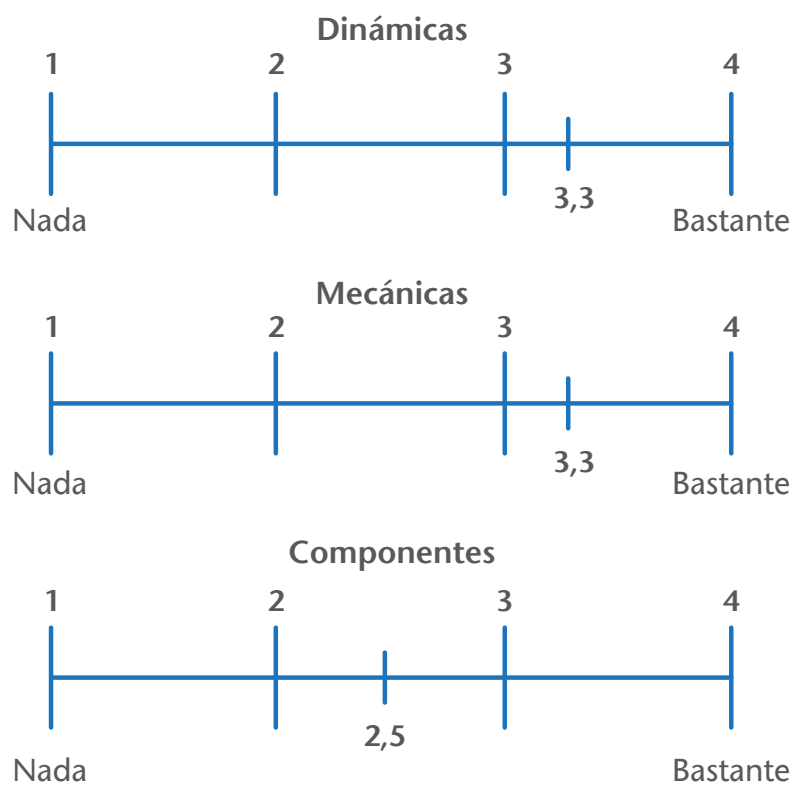

Fuente: elaboración propia.

Desde la perspectiva del estudiante, la intervención significó una integración elevada de las dinámicas y mecánicas de la clase. Con un resultado de 3,3 (entre las respuestas Regular y Bastante) para ambos rubros, los estudiantes coinciden con nuestra perspectiva acerca de la influencia de la propuesta didáctica en los elementos lúdicos que componen estas categorías. Cabe señalar que los resultados marcados con un asterisco ( $\left.{ }^{*}\right)$ en la Tabla 7 -que corresponden a los casos en donde el estudiante marcó la casilla Sin opinión- no fueron considerados para el promedio general, pero es importante observar que la mayoría de las respuestas implicadas correspondía a elementos lúdicos que, desde nuestra perspectiva, estuvieron ausentes en nuestra práctica docente, por ejemplo: el avatar, los huevos de Pascua, las insignias y las colecciones. Con esta información, podemos afirmar que tanto nuestras observaciones como la perspectiva del estudiante coincidieron en lo que se refiere a todos los elementos que intervinieron en la práctica docente. Ambas perspectivas estiman que la categoría que se trabajó menos en la propuesta didáctica fue la de los componentes de gamificación. En la Figura 4, podemos ver que se obtuvo un resultado de 2,5 en el nivel de percepción del estudiante acerca de los componentes, es decir, una implicación intermedia (entre las respuestas Poco y Regular).

En resumen, tanto en el análisis cualitativo como en el cuantitativo se tuvieron resultados similares respecto de la percepción de los beneficios de la gamificación. Esta intervención supuso la mejora de la participación e implicación de los estudiantes en las actividades de la materia, el desarrollo de un entorno innovador e interesante y el fortalecimiento de la relación estudiantil y de las habilidades interpersonales. Comparando los resultados de esta investigación con otras del mismo ámbito, hemos encontrado resultados similares. Por ejemplo, temíamos que los estudiantes se centraran más en las recompensas que en el desarrollo de las competencias de la materia; no obstante, sucedió todo lo contrario, pues las transacciones finales y los premios a los que podían acceder tomaron menos relevancia en la intervención, tal como lo exponen Gil y Prieto (2020, p. 118). Además, consideramos que nuestra intervención podría ser enriquecida para aumentar en mayor medida los resultados positivos, ya sea mediante la diversificación de medios para obtener puntos de compra (Corchuelo, 2018, p. 41) o con la creación de nuevas actividades que integren elementos poco trabajados (componentes) en esta experiencia didáctica.

\section{Conclusiones}

En definitiva, consideramos que la transformación de la práctica educativa mediante la gamificación tuvo un efecto positivo en la participación y realización de las actividades de la materia Desarrollo de la Expresión Escrita en Francés II. Tanto las 
ISSN 0123-1294 | e-ISSN 2027-5358 | Educ.Educ. Vol. 23. No.4 | Noviembre-enero de 2021 | pp. 611-633

Universidad de La Sabana | Facultad de Educación

Tabla 7. Percepción del estudiante sobre la gamificación

\begin{tabular}{|c|c|c|c|c|c|c|}
\hline \multirow[b]{2}{*}{ Gamificación } & \multicolumn{2}{|c|}{ Dinámicas } & \multicolumn{2}{|c|}{ Mecánicas } & \multicolumn{2}{|c|}{ Componentes } \\
\hline & 20 & Promedio & 32 & Promedio & 60 & Promedio \\
\hline Estudiante 1 & 15 & 3 & 20 & 2,5 & 27 & 1,8 \\
\hline Estudiante $2^{*}$ & 20 & 4 & 27 & 3,3 & 29 & $2,6^{*}$ \\
\hline Estudiante 3 & 18 & 3,6 & 30 & 3,7 & 47 & 3,1 \\
\hline Estudiante $4^{*}$ & 16 & 3,2 & 23 & 2,8 & 25 & $2,5^{*}$ \\
\hline Estudiante 5 & 15 & 3 & 23 & 2,8 & 40 & 2,7 \\
\hline Estudiante $6^{*}$ & 18 & 3,6 & 27 & 3,3 & 34 & $2,4^{*}$ \\
\hline Estudiante 7 & 18 & 3,6 & 30 & 3.7 & 30 & 2 \\
\hline Estudiante $8^{*}$ & 10 & $3,3^{*}$ & 25 & $3,5^{*}$ & 31 & $2,4^{*}$ \\
\hline Estudiante 9 & 17 & 3,4 & 25 & 3,1 & 46 & 3,1 \\
\hline Estudiante 10* & 17 & 3.4 & 27 & $3,8^{*}$ & 40 & $3,1^{*}$ \\
\hline Estudiante $11^{*}$ & 18 & 3,6 & 21 & $3,5^{*}$ & 31 & $2,4^{*}$ \\
\hline Estudiante 12 & 12 & 2,4 & 24 & 3 & 29 & $2,2^{*}$ \\
\hline Estudiante 13 & 16 & 3,2 & 28 & 3,5 & 33 & 2,2 \\
\hline Promedio del grupo & & 3,3 & & 3,3 & & 2,5 \\
\hline
\end{tabular}

*Casos donde el estudiante marcó la casilla "Sin opinión".

Fuente: elaboración propia.

actividades consideradas poco interesantes como aquellas que ya eran llamativas para los estudiantes tuvieron un efecto positivo mediante la gamificación. En general, se desarrolló su motivación intrínseca y extrínseca, mostraron mayor apertura a la realización de las actividades propuestas y su participación e implicación en ellas aumentó considerablemente. Desde la perspectiva del estudiante, se percibieron cambios significativos y la gamificación tuvo un impacto importante en el desarrollo de las competencias del programa. Por nuestra parte, podemos decir que se mejoró la comunicación, se desarrollaron las habilidades sociales del grupo y disminuyó el esfuerzo e intervención del profesor para motivar a los estudiantes. El entorno favorecido por los medios lúdicos permitió crear instrucciones y dinámicas que aumentaron el interés de los estudiantes por las actividades y trabajos de la materia en cuestión.
En síntesis, podemos destacar que, además que la gamificación no afecta negativamente el enfoque general de enseñanza ni los objetivos de aprendizaje, esta práctica educativa implica un cierto grado de esfuerzo creativo por parte del profesor en el rediseño de secuencias educativas congruentes y elementos que abonen a las dinámicas, mecánicas y componentes de gamificación. Además, esta perspectiva didáctica supone también mayor implicación del docente en la búsqueda de diversificación de actividades, así como en la atención a las características y efectos de esta práctica lúdica mediante un seguimiento continuo de los resultados de gamificación (puntos y clasificaciones) a los que se suman las competencias por desarrollar en el diseño curricular.

Para concluir, creemos que este estudio representa una contribución a las ciencias de la educación y, de manera precisa, a la didáctica, pues la gamifica- 
ción es todavía un tema que suscita interés y opiniones encontradas en cuanto a la pertinencia de su integración en el aula. Este trabajo nos ha permitido tener un panorama claro de los posibles beneficios e inconvenientes de una experiencia de gamificación en el contexto de enseñanza-aprendizaje del francés. No obstante, consideramos también que este aporte es solo una pequeña contribución que abona a la comprensión del objeto de estudio y se limita a un contexto muy particular. Dicho esto, nos parece necesario que surjan otros trabajos de investigación que coadyuven en el análisis y comprensión de dicha estrategia didáctica con el objetivo de profundizar en las implicaciones de esta en otros contextos educativos, pues sabemos que todo resultado de una intervención educativa dependerá en gran medida de los actores, de sus relaciones y del entorno en cual se desarrolla.

\section{Referencias}

Alejaldre, L. y García, A. (2015). Gamificar: el uso de los elementos del juego en la enseñanza del español [Ponencia]. Congreso La cultura hispánica: de sus orígenes al siglo XXI, Burgos, España. https://cvc.cervantes.es/ ensenanza/biblioteca_ele/aepe/congreso_50.htm

Aleksandrova, D. (2017). La motivación en clase de ELE [Ponencia]. Encuentro de Profesores de Español, Sofía, Bulgaria. https://cvc.cervantes.es/ensenanza/biblioteca_ele/publicaciones_centros/sofia_2017.htm

Aparicio, D., Torres-Barreto, M. y Álvarez-Melgarejo, M. (2018). Competencias ciudadanas desde un enfoque de gamificación. HAL-ArchivesOuvertes.fr. https://hal.archives-ouvertes.fr/hal-01952203/document

Barrios, E. (1997). Motivación en el aula de lengua extranjera. Revista de Investigación e Innovación en la Clase de Idiomas, 9, 17-30. https://dialnet.unirioja.es/servlet/articulo?codigo $=153504$

Briceño, E., Aguilar, R., Díaz, J. y Ucán, J. (2019). Gamificación para la mejora de procesos en ingeniería de software: Un estudio exploratorio. Revista Electrónica de Computación, Informática, Biomédica y Electrónica, 8(1), 1-20. http://recibe.cucei.udg.mx/ojs/index.php/ReCIBE/article/view/123/97

Borrás-Gené, O. (2015). Fundamentos degamificación. Universidad Politécnica de Madrid. https://doi.org/10.13140/ RG.2.1.3807.9848

Castro, F. (2015). Motivación y esfuerzo en la adquisición de lenguas extranjeras. En Y. Morimoto, L. Pavón, V. María y R. Santamaría (eds.), La enseñanza de ELE centrada en el alumno (pp. 213-220.). Asociación para la Enseñanza del Español como Lengua Extranjera. https://dialnet.unirioja.es/servlet/libro?codigo=581025

Corchuelo-Rodríguez, C. (2018). Gamificación en educación superior: experiencia innovadora para motivar estudiantes y dinamizar contenidos en el aula. Revista Electrónica de Tecnología Educativa, 63, 29-41. https:// doi.org/10.21556/edutec.2018.63.927

Díaz-Barriga, F. (2006). Enseñanza situada: vínculo entre la escuela y la vida. McGraw-Hill. 
Fabila, A., Minami, H. e Izquierdo, J. (2013). La escala de Likert en la evaluación docente: acercamiento a sus características y principios metodológicos. Perspectivas Docentes, 50, 31-40. https://dialnet.unirioja.es/servlet/ articulo?codigo $=6349269$

García, J. (2011). Modelo educativo basado en competencias: importancia y necesidad. Actualidades Investigativas en Educación, 11(3), 1-24. https://www.redalyc.org/pdf/447/44722178014.pdf

Gil-Quintana, J. y Prieto, E. (2020). La realidad de la gamificación en educación primaria. Estudio multicaso de centros educativos españoles. Perfiles Educativos, 42(168), 107-123. https://doi.org/10.22201/iisue.24486167e.2020.168.59173

González, C. (2019). Gamificación en el aula: ludificando espacios de enseñanza-aprendizaje presenciales y espacios virtuales. [Documento web] https://doi.org/10.13140/RG.2.2.34658.07364

Hélou, C. y Lantheaume, F. (2008). Les difficultés au travail des enseignants. Recherche et Formation, 57, 65-78. https://doi.org/10.4000/rechercheformation. 833

Hernández, R., Fernández, C. y Baptista, M. (2014). Metodología de la Investigación. McGraw-Hill.

Lee, J. y Hammer, J. (2011). Gamification in education: What, how, why bother? Academic Exchange Quarterly, 15(2), 1-4. https://dialnet.unirioja.es/servlet/articulo?codigo=3714308

Matas, A. (2018). Diseño del formato de escalas tipo Likert: un estado de la cuestión. Revista Electrónica de Investigación Educativa, 20(1), 38-47. https://doi.org/10.24320/redie.2018.20.1.1347

Monguillot, M., González, C., Zurita, C., Almirall, L. y Guitert, M. (2015). Play the Game: gamificación y hábitos saludables en educación física. Apunts: Educación Física y Deportes, 119, 71-79. https://dialnet.unirioja.es/ servlet/articulo?codigo $=5068163$

Olvera-Lobo, M. y Gutiérrez-Artacho, J. (2016). Gamificación para la adquisición de competencias en la educación superior: el caso de la Traducción e Interpretación. En G. Padilla (Ed.), Aulas virtuales: Fórmulas y prácticas (pp. 199-216.). McGraw-Hill.

Oliva, H. (2016). La gamificación como estrategia metodológica en el contexto educativo universitario. Realidad y Reflexión, 44, 29-47. https://doi.org/10.5377/ryr.v44io.3563

Ortiz-Colón, A., Jordán, J. y Agredal, M. (2018). Gamificación en educación: una panorámica sobre el estado de la cuestión. Educação e Pesquisa, 44, 1-17. http://dx.doi.org/10.159o/s1678-4634201844173773

Parente, D. (2016). Gamificación en la educación. En R. Contreras y J. Eguia (eds.), Gamificación en aulas universitarias (pp. 11-21). Universitat Autònoma de Barcelona.

Rodríguez, J. (2016). Aprendizaje basado en juegos. En P. Lavado y V. Lacambra (eds.), IX Jornadas Nacionales de Ludotecas. Ponencias y comunicaciones. Juegos y juguetes en la vida social (pp. 139-152). Comarca de la Sierra de Albarracín. 
Romero-Rodríguez, L., Torres-Toukoumidis, Á. y Aguaded, I. (2017). Ludificación y educación para la ciudadanía. Revisión de las experiencias significativas. Educar, 53(1), 109-128. https://www.raco.cat/index.php/Educar/ article/view/317273

Sánchez-Rivas, E. y Pareja-Prieto, D. (2015). La gamificación como estrategia pedagógica en el contexto escolar. En J. Ruiz-Palmero, J. Sánchez-Rodríguez y E. Sánchez-Rivas (eds.), Innovaciones con tecnologías emergentes. Universidad de Málaga.

Sánchez, M. (2007). Buenas prácticas en la creación de Serious Games (Objetos de Aprendizaje Reutilizables). [Ponencia] IV Simposio Pluridisciplinar sobre Diseño, Evaluación y Desarrollo de Contenidos Educativos Reutilizables, 19 a 21 de septiembre, Bilbao. http://ceur-ws.org/Vol-318/Sanchez.pdf

Séraphin, A. (2016). L'enseignant face à la difficulté de la classe: Capacité à agir et décrochage enseignant. Questions Vives, 25, 1-20. https://doi.org/10.4000/questionsvives.1942

Trejo, H. (2019). Recursos tecnológicos para la integración de la gamificación en el aula. Tecnología, Ciencia y Educación, 13, 75-117. https://www.revistasocitec.org/index.php/TCE/article/view/285

Werbach, K. y Hunter, D. (2012). For the win: How game thinking can revolutionize your business. Wharton Digital Press. 